\title{
Clinical utility and patient considerations in the use of ofatumumab in chronic lymphocytic leukemia
}

\author{
Anna Maria Frustaci \\ Alessandra Tedeschi \\ Paola Picardi \\ Roberto Cairoli \\ Marco Montillo
}

Department of Hematology, Niguarda Cancer Center, Niguarda Ca' Granda Hospital, Milan, Italy
Correspondence: Marco Montillo Department of Hematology, Niguarda Cancer Center, Niguarda Ca' Granda Hospital, Piazza Ospedale Maggiore 3, Milan 20162, Italy

$\mathrm{Tel}+390264444074$

Fax +390264443263

Email marco.montillo@ospedaleniguarda.it
This article was published in the following Dove Press journal:

Biologics: Targets and Therapy

18 September 2015

Number of times this article has been viewed

\begin{abstract}
Treatment aim for chronic lymphocytic leukemia has been radically changed over the past years from providing only a palliative approach to reaching disease eradication and improving survival. Ofatumumab is a monoclonal humanized antibody with peculiar in vitro and in vivo properties, at present approved for double fludarabine and alemtuzumab refractory chronic lymphocytic leukemia. Its efficacy in this subset of patients, who typically have an unfavorable prognosis, facilitated its use in different Phase II and III trials. Ofatumumab as single agent or combined with chemotherapeutic or biologic agents, led to sundry results in the setting of both previously treated or untreated patients. Its role in maintenance therapy is also under investigation. Further advances concerning ofatumumab administration as first line therapy in combination with chlorambucil, came recently from the COMPLEMENT 1 study. Results from this trial will open the door to new perspectives of its use in treatmentnaïve patients. Ofatumumab was well tolerated in almost all the studies, with the main adverse events relating mostly to infusion reaction. Hematologic toxicity, especially neutropenia, was also common. A significant improvement in patients' quality of life was reported following ofatumumab treatment and this was mainly due to its effect on constitutional symptoms. Nevertheless, some concerns remain regarding the long-term efficacy of the drug in terms of response duration and survival. The real strength of this drug needs to be confirmed by further studies and direct comparative trials.
\end{abstract}

Keywords: ofatumumab, chronic lymphocytic leukemia, refractory, alemtuzumab, fludarabine, high risk

\section{Introduction}

Treatment of chronic lymphocytic leukemia (CLL), has represented a curious paradigm over the past decade, as therapy aim has radically changed from providing pure symptom palliation, to reaching complete and even molecular remission and improving survival.

Considering the fact that the majority of CLL population is represented by a median age of 72 years $^{1}$ and that most of the patients will encounter multiple lines of therapy during disease course, the need for drugs other than chemotherapy has become a priority during the last few years.

The advent of monoclonal antibodies (MoAb), in view of their fully biological and targeted mechanism of action, represents important progress in this direction.

The first of this category to obtain the US Food and Drug Administration (FDA) regular approval for the treatment of first or subsequent line of CLL was alemtuzumab, a humanized MoAb that recognizes CD52. Alemtuzumab induced responses in 34\% 
of fludarabine refractory patients and showed efficacy also in those with TP53 abnormalities..$^{2,3}$ Moreover, as a singleagent it demonstrated efficacy in naïve patients too, with a significant improvement in the overall response rate (ORR), complete response rate, and progression free survival (PFS) when compared to chlorambucil. ${ }^{4}$ Major alemtuzumab limits are represented by lack of efficacy in bulky disease, deep immunosuppression, and risk of opportunistic infections. ${ }^{5}$

CD20 is a cell surface molecule, highly expressed in B-cells. Its role is mainly represented in the production of T-cell-independent antibody response and B-lymphocyte activation. CD20 is exclusively expressed in B-cells, and the antigen is not shed or internalized in the remaining cells. Moreover, CD20 is not present on stem cells, precursor cells, or plasma cells. ${ }^{6}$ These characteristics make CD20 a suitable therapeutic target for B-cell malignancies and its related therapeutic application opened the door for a radical change in CLL treatment.

Rituximab is a chimeric anti CD20 MoAb. Direct signaling, complement dependent cellular cytotoxicity (CDC), and antibody dependent cellular cytotoxicity, all appear to play a role in its efficacy. ${ }^{7}$

Obviously, the possibility to use a single biological drug for the treatment of CLL, represents a desirable hope for a chronic disease. In CLL, however, CD20 expression is dim and this could explain the weak results obtained with rituximab in monotherapy in relapsed or refractory cases. ${ }^{8,9}$ In this setting, a partial response of $10 \%-15 \%$ could be improved to $30 \%$ with the adjustment of rituximab dose. ${ }^{10}$ The same schedules, applied to another subset of untreated population, led to an increased response rate of $58 \% .^{11}$

As a natural consequence of the enhanced activity demonstrated in non-Hodgkin lymphomas (NHL) by the addition of rituximab to chemotherapy, ${ }^{12-14}$ the next step was the addition of rituximab to chemotherapy also in CLL.

The combination of rituximab and fludarabine plus cyclophosphamide (FCR), has been established as the gold standard regimen for young and fit CLL patients due to its significant improvement of PFS and overall survival (OS) in this category both for previously treated and untreated patients. ${ }^{15,16}$

Moreover, considering the need to cover a more representative elderly CLL population with comorbidities, further studies tested the efficacy and tolerability of rituximab combined with more manageable chemotherapeutic agents such as chlorambucil, pentostatin, cladribine or bendamustine. ${ }^{17-23}$

Despite a clear benefit of rituximab addition, in terms of response quality and PFS, all patients are expected to relapse after a rituximab containing regimen. Therefore, there still remains a significant unmet need in CLL.

Furthermore, given the well-known genetic and clinical variability of CLL, a limited group of patients can be defined as "high risk" and will be mostly doomed to fail under standard therapeutic approaches. Those categories are represented by patients with unfavorable cytogenetic or molecular features, as well as patients who are resistant to a fludarabine or alemtuzumab based therapy. ${ }^{24-26}$

Ofatumumab is a fully human IgG1-type anti CD20 $\mathrm{MoAb}$. Its ability to bind to both the small and large loop of the membrane antigen CD20 allows a prolonged dissociation rate. Compared to rituximab, ofatumumab is able to produce a greater CDC activity with a similar antibody dependent cellular cytotoxicity activity, especially in low CD20-expressing CLL cells. ${ }^{27-29}$ Furthermore, complement cascade seems to be easily activated by ofatumumab due to its binding avidity to $\mathrm{C} 1 \mathrm{q} .{ }^{30}$

Lastly, ofatumumab demonstrated to be effective in purging CD20 positive CLL cells even in those patients resistant to rituximab, both in vitro and in vivo. ${ }^{31}$

Such a promising drug profile led to a high expectancy related to ofatumumab offered to those patients with worst clinical disease features, in particular fludarabine-refractoriness and those carrying a $17 \mathrm{p}$ deletion. In fact, despite the demonstrated efficacy of alemtuzumab offered to this population, this anti-CD52 antibody is unable to produce results in patients with bulky disease. .,32 $^{5}$

\section{Ofatumumab in relapsed/ refractory CLL Single agent ofatumumab}

The first clinical study evaluating ofatumumab efficacy and tolerability was performed by Coiffier et al, ${ }^{33}$ who reported the experience with 33 patients in the Hx-CD20-402 dose escalation study. Three cohorts of patients were selected with different ofatumumab dosages (maximum dose $500 \mathrm{mg}$ in cohort A; 1,000 mg in B, and 2,000 $\mathrm{mg}$ in C), established based on previous drug experience in follicular lymphoma. Patients received four weekly infusions with a starting dose of 100,300 , and $500 \mathrm{mg}$ respectively.

Although most of the study population belonged to a lowintermediate Rai-Binet risk stage (84\% Rai I-II; 88\% Binet A-B), the majority of them were heavily pretreated with a median number of three prior therapies.

Ofatumumab used as a single agent, showed efficacy with 13/14 responding patients belonging to cohort C. Median PFS resulted approximately 3 months, but with a median time to next treatment of 1 year. Objective responses were rapid 
with a large portion of patients on the 2,000 $\mathrm{mg}$ schedule, showing halving of lymph node size from week 4 and then gradually progressing. An encouraging improvement in baseline cytopenia was also observed. The lack of biologic patient characterization probably represented a main limitation of the study, particularly considering proof of a drug's in vitro activity on P53 mutant CLL cells.

Based on those encouraging results, a dose of 2,000 mg was chosen for the subsequent Phase II trial (406 Study) with ofatumumab monotherapy. After a planned interim analysis on 138 patients, final results from the study have recently been published. ${ }^{34,35}$ Irrespective of the prior use of rituximab, a large cohort of heavily pretreated CLL patients were categorized as fludarabine-alemtuzumab refractory (FA-ref; 95 at the final analysis) and bulky-fludarabine refractory (BF-ref; 112 at the final analysis). Only $51 \%$ of patients could complete the planned 12 infusions even though the majority received at least eight doses. Ofatumumab alone showed impressive results in terms of response rate and survival, when compared with the historical data regarding both intensive regimen or other MoAb alone in fludarabine refractory patients. In the last report from the final analysis, $49 \%$ and $43 \%$ of responses were observed in FA-ref and BF-ref, respectively. Ofatumumab demonstrated to be active in cytopenias with a large proportion of cases improving baseline hemoglobin and platelet values. Even in those subjects who didn't obtain an objective response, a significant improvement of constitutional symptoms was recorded. As confirmed in the final results, among all the examined baseline features, only those carrying $17 \mathrm{p}$ deletion obtained a significant lower ORR (30\% vs $53 \%$ in $17 \mathrm{p}$ deletion; $P=0.0055)$. According to the interim analysis, OS was significantly increased ( $\geq 10$ months) in responders with a median not reached in both FA- and BFref groups. Median response duration resulted in 7.1 and 5.6 months respectively in FA- and BF-ref. Finally, in the updated results, demographic and disease baseline features combined with ofatumumab pharmacokinetic parameter values were examined by univariable and multivariable analyses. These results demonstrated a main relevance of disease burden and individual factors, rather than ofatumumab exposure, on clinical outcomes. Authors conclude that patients with a high disease burden may benefit from ofatumumab dose intensity and maintenance strategy.

A retrospective analysis from the same authors, ${ }^{36}$ evaluated the impact of previous rituximab treatment on the response achievement to ofatumumab. Among 206 patients, 117 received prior therapy with rituximab either alone or in combination. ORR results were $43 \%$ for those who previously received rituximab and 53\% for those who were rituximab naïve. A longer median PFS was described in rituximab naïve cases with no significant difference in median OS. A longer time from last rituximab scheme (irrespective of combination, response status or time to progression [TTP]), resulted in significant survival improvement. In summary, ofatumumab was effective irrespective of prior rituximab exposure.

\section{Combination therapy}

Recently, GIMEMA reported the results of a multicenter Phase II study with ofatumumab used in combination with bendamustine in pretreated patients. ${ }^{37}$ Among 47 patients with a median of 66 years, $17(35 \%)$ were aged $\geq 70$; eleven $(22 \%)$ presented an unfavorable TP53 disruption status detected either by cytogenetic or molecular analysis. Most of them were previously treated with a fludarabine-based regimen. ORR was $72 \%$ with $17 \%$ of complete remissions (CRs). Five patients (11\%) progressed. After a median follow-up of 24.2 months median OS and PFS results were $83.6 \%$ and $49.6 \%$ respectively with a median PFS of 23.6 months. Even when combined with chemotherapy, treatment was well tolerated by almost all patients completing the six planned courses. Five disease-related deaths occurred after therapy (CLL progression in three cases, Richter syndrome in two).

The Phase II study OMB1 15991, ${ }^{38}$ reported the use of the same combination both in previously treated and untreated cases. Among those who relapsed, median age was 68 years, $41 \%$ of cases presented an adverse $17 \mathrm{p} / 11 \mathrm{q}$ deletion and $72 \%$ an unmutated IgHV status. Overall response result was 74\%, with $11 \%$ CRs. No patients reached molecular negativity. Median duration of the study ( 8.7 months) was too short to interpret follow-up data.

A smaller experience with ten cases was reported by Ujjani et $\mathrm{al}^{39}$ with an inferior ORR of $40 \%$. This trial was prematurely discontinued due to an expected excess of toxicity including development of three cases of Richter syndrome.

The use of ofatumumab combined with nonchemotherapeutic agents has also been investigated. The association of ofatumumab administered at a lower dose of 1,000 mg, with high dose corticosteroids such as methylprednisolone $(\mathrm{mPDN})^{40}$ or dexamethasone $(\mathrm{DMS})^{41}$ was tested in 21 and 33 patients respectively. One third of the patients in the study on mPDN and $42 \%$ on DMS presented an adverse cytogenetic feature (17p or 11q deletion). Despite a considerable response rate, only a low percentage of patients obtained a CR. PFS result was 10 months in both studies. A favorable median OS of 34 months was achieved 
in association with DMS while it was not reached after 31 months with mPDN.

Lenalidomide with its complex immunomodulatory action and synergic action when combined with rituximab, ${ }^{42}$ also represents a potentially tempting association drug. Its use in combination with ofatumumab was feasible and led to a response in half of the 21 subjects enrolled in a Phase II trial. ${ }^{43}$ Such a response rate seems more consistent considering the elevated presence of patients with unfavorable pretreatment characteristics (52\% had a Rai IV stage, $86 \%$ an unmutated IgHV status, $29 \%$ a 17 p deletion, and $33 \%$ an 11 q deletion). A median survival of 21.5 months result was satisfactory and authors continue to speculate regarding the possible use of longer lenalidomide maintenance or an ofatumumab dose intensity.

\section{Observational retrospective studies}

Outside of clinical trials, efficacy and tolerability of ofatumumab were retrospectively analyzed in different reports. Despite the limits of a retrospective analysis, these reports avail themselves of real life experiences.

Moreno et al, ${ }^{44}$ reported the data from 103 CLL patients treated with ofatumumab outside of clinical trials. Median age was relatively young and the majority showed an advanced symptomatic disease. Twenty-two among 53 patients were categorized as FA-ref; 22/50 as BF-ref. Median number of administered cycles was nine; in $13 \%$ of cases, another agent was added to ofatumumab (mostly chlorambucil or corticosteroids). Overall response results were $22 \%$ with $4 \%$ of CR. None of those classified as a high cytogenetic risk reached a durable response. After a median follow-up of 9.4 months, median PFS and OS were 5 and 11 months respectively. Advanced clinical stage, treatment failure, and prior fludarabine refractoriness was significantly associated with a shorter OS. This series can be reasonably considered as a high risk, mainly due to the elevated number of patients with fludarabine refractory disease status, significant number of prior lines of therapy (median four), and presence of patients who received allografts (13\%). Applied to "real life" ofatumumab as monotherapy obtained half of the response rate previously reported by Coiffier et $\mathrm{al}^{33}$ and Wierda et $\mathrm{al}^{34}$ was confirmed to be effective in improving cytopenias; and showed similar PFS and OS to those reported previously.

Data from 27 patients from the UK compassionate program had been extrapolated before ERIC report. ${ }^{45}$ ORR (48\%) reproduced data from Wierda et al, ${ }^{34}$ but with a disappointing time to next treatment of 5.5 months. Four among six cases carrying a $17 \mathrm{p}$ deletion obtained a response; interestingly, in two patients achieving a $\mathrm{CR}$, a prolonged response duration of 18 months was seen. As previously reported, ofatumumab led to a meaningful improvement of hemoglobin/platelets values.

Lastly, data from French Early Access Program ${ }^{46}$ were published, from a heavily pretreated (median six lines of previous therapy) cohort of patients. In the French series, responses were similar to those previously reported, while survival data were slightly inferior. In 17 p cases, ofatumumab was shown to achieve sustained responses.

\section{Ofatumumab in treatment-naïve patients Single agent ofatumumab}

While FCR combination is widely approved as the best initial therapy for young, fit patients, ${ }^{15,16}$ there is still a significant unmet need regarding older fit and unfit population. Moreover, not all the patients are able to tolerate FCR and a subset of high risk population is destined to fail with this combination. ${ }^{47,48}$

Due to its pharmacodynamic properties, its ability to overcome rituximab resistance, and its low toxicity profile, ofatumumab alone or in combination could represent a potentially effective treatment in different subsets of CLL populations.

Ofatumumab administered with the classical single agent schedule, has been offered as first line treatment in elderly or young patients who declined fludarabine. ${ }^{49}$ First response evaluation was performed after 8 weeks. Patients with progressive disease were excluded from the protocol, while the rest were considered eligible to receive ofatumumab as maintenance therapy for 2 years. At the interim analysis on 42 subjects, all but one patient were included in the maintenance phase with a partial response (PR) in $13(44 \%)$ cases, and stable disease in the remaining 16 (53\%).

Preliminary results from ofatumumab as first line treatment on an intentionally selected unfit population were presented by MD Anderson Cancer Center (MDACC).$^{50}$ Patients with other malignancies were also included. Ofatumumab was administered at a lower dosage of $1,000 \mathrm{mg}$ to the first eight patients, then increased to 2,000 mg. Eighteen patients participated in the study; nine among the 13 evaluable, obtained a response with three CRs. At a median follow-up of 24 months, six patients remained progression-free and seven needed further treatment after a median of 15 months.

Again MDACC investigated the potential role of premature ofatumumab therapy used with the aim to delay time to 
first chemo-immunotherapy. ${ }^{51}$ Only patients not presenting an International Workshop on Chronic Lymphocytic Leukemia/ National Cancer Institute Working Group (IWCLL/NCI-WG) treatment indication and carrying an adverse laboratory or biological feature could be included in the study. Median age was 59 years old; half of the cases presented an unmutated IgHV status, and $34 \%$ an unfavorable $17 \mathrm{p}$ or $11 \mathrm{q}$ deletion. Eight ofatumumab infusions were administered weekly. After a median follow-up of 4.7 months, one third of the 18 evaluable patients presented with stable disease, the remaining 12 reached a response. Three patients progressed at 18.8, 14.1, and 3.2 months from start of treatment. Interestingly, none of the patients with $17 \mathrm{p} / 11 \mathrm{q}$ deletion progressed. Authors speculated on the potential ofatumumab benefit of firsttreatment delay.

\section{Combination therapy}

Promising results were provided by a Phase II study exploring the combination of alemtuzumab and ofatumumab. ${ }^{52}$ At the interim analysis, 31 patients with a median age of 63 years were evaluated. Protocol was amended after two cases (one fatal) of prolonged cytopenia and patients were allowed to prematurely withhold alemtuzumab or ofatumumab as soon as they achieved minimal residual disease (MRD) marrow negativity, complete nodal remission or in case of low marrow cellularity. Response depth was highlighted by a third of patients meeting early response criteria after a median of 10 weeks' alemtuzumab and five ofatumumab administrations. All patients but one reached a response, with $42 \% \mathrm{CR}$. Almost half of the cases (13 in CR and four in PR), achieved MRD negative status.

In 2010, Hallek et al demonstrated with the Phase III randomized CLL8 trial, a clear advantage in terms of response rate achievement and survival, of chemo-immunotherapy with FCR in comparison with fludarabine and cyclophosphamide (FC) chemotherapy in CLL treatment-naïve patients. ${ }^{16}$

Taking into account those results, the first study combining ofatumumab with FC backbone as first line treatment, was conducted by Wierda et al as a Phase II trial. ${ }^{53}$ Two cohorts of patients were treated with FC combination added to ofatumumab 500 or $1,000 \mathrm{mg}$ for up to six cycles. Complete remission rate (CRR) was the primary end point of the trial and did not differ between the $500 \mathrm{mg}(32 \%)$ and 1,000 $\mathrm{mg}$ $(50 \%)$ cohort with a statistical trend in favor of the latter.

The trial enrolled a CLL population significantly younger than usual (median age 56 years). Fludarabine, cyclophosphamide, and ofatumumab (FCO) combination, even with ofatumumab $1,000 \mathrm{mg}$, failed to demonstrate a clear superiority compared to historical data from FCR, in terms of both CRR ( $50 \%$ vs $44 \%$ ) and ORR (73\% vs $90 \%)$. Patients with $17 \mathrm{p}$ deletion, (8/61) and also those who could benefit by the addition of ofatumumab, showed a similar poor outcome as those with the same chromosomal features treated with FCR.

Authors correlate those results with the higher risk profile of their population in respect to CLL8 study. Interestingly, the use of the higher ofatumumab dosage of $1,000 \mathrm{mg}$, did not translate to an excess of either hematological or nonhematological toxicity.

With the aim of reducing chemo-related toxicity observed when an MoAb with FC was applied, two Phase II trials explored the combination of pentostatin (a purine analog with a higher tolerability compared to fludarabine) with cyclophosphamide and ofatumumab (PCO). ${ }^{54,55}$ PCO combination, first described by Shanafelt et al in 48 patients, ${ }^{54}$ showed an overall response of $96 \%$ with almost half of the responders presenting complete remission. Seven among the 38 examined cases, obtained a negative MRD. These data are comparable to those reported with the same pentostatin combination added to rituximab (PCR); nevertheless PCO led to a longer time to retreatment with $86 \%$ of patients free from a new therapy at 24 months $(68 \%$ at 24 months with combination of PCR). Another Phase II trial using the same PCO schedule adopted by the Mayo Clinic, was conducted by a north Italian group for CLL. ${ }^{55}$ This was targeted specifically to the elderly who represented the group mainly exposed to the toxicity of FCR. Also when used in a population with a median age of 72 years, PCO demonstrated efficacy inducing $89 \%$ response with 51\% complete remission. Moreover, 19 among the 24 tested for MRD, resulted in an MRD negative status. As previously reported, the attainment of a profound response translated to a longer PFS, estimated as $74 \%$ at 24 months.

The older population was also the target of the COMPLEMENT 1 trial $^{56}$ which compared chlorambucil added to ofatumumab with chlorambucil alone as front line therapy in patients not suitable for fludarabine-based treatment. This large Phase III trial randomized 447 patients with a median age of 69 . The arm of chemo-immunotherapy compared to the arm of chlorambucil alone showed a significantly higher CRR (12\% vs 1\%), and longer PFS (22 vs 13 months). Only patients in CR were tested from peripheral blood for minimal residual disease and the result was negative in $8 \%$ of patients treated with ofatumumab vs a single case in the chemotherapy arm. Moreover, patients with $17 \mathrm{p}$ deletion assigned to chlorambucil plus ofatumumab showed a not 
statistically significant improvement in PFS in comparison with those with $17 \mathrm{p}$ deletion in chlorambucil arm. With a median follow-up of 29 months, median survival has not yet been reached in both groups. It is however important to note that chemo-immunotherapy did not show an advantage in terms of survival compared to chlorambucil. Grade 3-4 adverse events (AEs) did not differ between the two populations.

Finally, OMB115991 study ${ }^{38}$ evaluated ofatumumab combined with bendamustine in previously untreated patients not suitable for a fludarabine-based treatment. In 44 patients with a median age of 62 years old, ORR resulted in $95 \%$ with $48 \%$ CRs. Notably, among those with a CR, 56\% reached MRD eradication. Median time to response was less than 1 month. After a median study duration of 8.5 months, follow-up data were not yet ready to be interpreted. All the details regarding the mentioned studies are summarized in Table 1.

\section{Maintenance}

Recent data from GEN416 study ${ }^{57}$ were aimed to inquire into the efficacy of ofatumumab retreatment and maintenance. Authors extrapolated a subset of 29 patients from the 406 Study. ${ }^{34}$ Patients who obtained at least a stable disease with the previous ofatumumab treatment, and then afterwards relapsed or progressed, were eligible for retreatment with eight weekly ofatumumab infusions; in those with disease control, monthly maintenance therapy up to 2 years was administered. It is important to note that patients who underwent a containing regimen during the time between treatment and retreatment, were not excluded from the study.

Table I Clinical trials in previously untreated patients

\begin{tabular}{|c|c|c|c|c|c|c|}
\hline Authors & Schedule & $\begin{array}{l}\text { Patient } \\
\text { number }\end{array}$ & $\begin{array}{l}\text { ORR (\%) } \\
\text { (CRR \%) }\end{array}$ & Follow-up (mo) & PFS (mo) & OS (mo) \\
\hline Flinn et al $2012^{49}$ & $\begin{array}{l}\text { O: } 300 \mathrm{mg} \text { DI; } 2,000 \mathrm{mg} \text { D8; } 2,000 \mathrm{mg} \text { weekly } \\
\text { for } 6 \mathrm{w} ; 2,000 \mathrm{mg} \text { monthly for } 4 \mathrm{mo}\end{array}$ & 42 & $44(0)$ & NR & NR & NR \\
\hline Ciccone et al $2014^{50}$ & $\begin{array}{l}\text { O: } 300 \mathrm{mg} \text { DI; } 2,000 \mathrm{mg} \text { D8; } \\
\text { DI5; D22 (Cl); } \\
2,000 \mathrm{mg} \text { monthly for } 12 \mathrm{mo} \text { (O: I,000 mg } \\
\text { for the first } 8 \text { patients) }\end{array}$ & 13 & $69(23)$ & 24 & $46 \%$ & $92 \%$ \\
\hline Jain et al $2013^{51}$ & O: 300 mg DI, I,000 mg weekly for 7 w & 18 & $67(17)$ & 7.6 & $83 \%$ & $100 \%$ \\
\hline Ma et al $2014^{52}$ & $\begin{array}{l}\text { Alem } 3 \text { times/w up to I } 8 \text { w } \\
\text { O: (from w3): } 300 \mathrm{mg} \mathrm{DI} ; \\
2,000 \mathrm{mg} \text { q } 2 \text { w doses week } 2 \text { to week } 8 \text { (early } \\
\text { interruption based on response assessment) }\end{array}$ & 31 & $97(42)$ & NR & $90 \%$ & $94 \%$ \\
\hline Wierda et al $201 I^{53}$ & $\begin{array}{l}\text { F: } 25 \mathrm{mg} / \mathrm{m}^{2} \mathrm{D} 2-4 \\
\mathrm{C}: 250 \mathrm{mg} / \mathrm{m}^{2} \mathrm{D} 2-4+ \\
\text { O: } 300 \mathrm{mg} \mathrm{DICI} \rightarrow 500 \mathrm{mg} \text { DIC2-6 vs } \\
\text { O: } 300 \mathrm{mg} \mathrm{DICI} \rightarrow \mathrm{I}, 000 \mathrm{mg} \text { DIC2-6 } \\
\text { q28 } 66 \text { cycles }\end{array}$ & $\begin{array}{l}31 \text { vs } \\
30\end{array}$ & $\begin{array}{l}77(32) \text { vs } \\
73(50)\end{array}$ & 8 & $\mathrm{nr}$ & $\mathrm{nr}$ \\
\hline Shanafelt et al $2013^{54}$ & $\begin{array}{l}\mathrm{P}: 2 \mathrm{mg} / \mathrm{m}^{2} \\
\mathrm{C}: 600 \mathrm{mg} / \mathrm{m}^{2} \\
\text { O: CI } 300 \mathrm{mg} \text { DI; I,000 mg D8 } \\
\text { C2-6 I,000 mg DI } \\
\text { q2I } \times 6 \text { cycles }\end{array}$ & 48 & $96(46)$ & 24 (living patients) & $\mathrm{nr}(\mathrm{TTR})$ & $\begin{array}{l}42 \text { patients alive } \\
\text { at } 24 \text { months }\end{array}$ \\
\hline Montillo et al $2015^{55}$ & $\begin{array}{l}\text { P: } 2 \mathrm{mg} / \mathrm{m}^{2} \\
\text { C: } 600 \mathrm{mg} / \mathrm{m}^{2} \\
\text { O: CI } 300 \mathrm{mg} \mathrm{DI} ; \mathrm{I}, 000 \mathrm{mg} \text { D8 } \\
\text { C2-6 I,000 mg DI } \\
\text { q2I } \times 6 \text { cycles }\end{array}$ & 47 & $89(5 \mathrm{I})$ & 22 & $\mathrm{nr}$ & $\mathrm{nr}$ \\
\hline Hillman et al $2015^{56}$ & $\begin{array}{l}\text { Chl: } 10 \mathrm{mg} / \mathrm{m}^{2} \mathrm{DI}-7 \mathrm{q} 28 \text { up to } 12 \text { cycles vs } \\
\text { O: } \mathrm{Cl} 300 \mathrm{mg} \mathrm{DI} ; 1,000 \mathrm{mg} \text { D8 } \\
\text { C2-6 I,000 mg DI + } \\
\text { Chl: } 10 \mathrm{mg} / \mathrm{m}^{2} \mathrm{DI}-7 \\
\text { q28 up to } 12 \text { cycles }\end{array}$ & $\begin{array}{l}226 \text { vs } \\
221\end{array}$ & $\begin{array}{l}69(1) \text { vs } \\
82(14)\end{array}$ & 29 & $\begin{array}{l}13 \text { vs } \\
22\end{array}$ & $\begin{array}{l}\mathrm{nr} \\
\mathrm{nr}\end{array}$ \\
\hline Offner et al $2014^{38}$ & $\begin{array}{l}\text { O: } 300 \mathrm{mg} \text { CIDI, I,000 mg CID8; I,000 mg } \\
\text { DIC2 to C6 } \\
\text { B: } 90 \mathrm{mg} / \mathrm{m}^{2} \mathrm{DI}-2 \mathrm{CI} \rightarrow 6\end{array}$ & 44 & $95(48)$ & 8.5 & NR & NR \\
\hline
\end{tabular}

Abbreviations: O, ofatumumab; Alem, alemtuzumab; F, fludarabine; C, cyclophosphamide; P, pentostatin; Chl, chlorambucil; B, bendamustine; ORR, overall response rate; CR, complete response; CRR, complete remission rate; PFS, progression free survival; OS, overall survival; mo, month(s); NR, not reported; nr, not reached; D, day; w, week(s); TTR, time to retreatment; $C$, cycle. 
Overall, $45 \%$ of cases showed a response with ofatumumab retreatment, mainly among the FA-ref group. Responses were rapid ( $\leq 2$ months in median) and durable (median 24.1 months). Response duration favorably compared with that of the 406 Study (6.8 months), while PFS and time to next treatment (TTNT) were comparable. Median survival results were 18 months in FA-ref and 11.3 months in BF-ref group. Considering that this population consisted of patients who lost or never obtained the initial response to ofatumumab, survival data reflect what was already highlighted in the final analysis from the 406 Study. In the 406 Study, indeed, median OS was significantly longer among responding patients compared with non-responders (FA-ref: 24.9 months vs 9.9 months and BF-ref 28.9 months vs 15.5 months [ $P=0.0154]$ ).

Finally, PROLONG is the first study specifically focused on the possible role of ofatumumab as maintenance therapy. ${ }^{58}$ A large cohort of 474 patients responding to a second or third line of treatment, were randomized to receive ofatumumab as maintenance therapy up to 2 years vs observation. The two groups were stratified according to number and type of prior therapies and the achieved response. After a median follow-up of 26 months, ofatumumab prolonged therapy achieved the primary end point, PFS being significantly longer than in the observation arm (28.6 months vs 15.2 respectively). This translated to a significant time of 10 months longer to next treatment in the maintenance group. Ofatumumab seemed to be an active and well tolerated treatment. Further information to establish its role as maintenance therapy is warranted.

\section{Toxicity}

Overall, ofatumumab demonstrated to be well tolerated both when used alone or in combination. Similarly to rituximab most of the reported AEs in different studies are infusion-related and include chills, fever, rash, fatigue, gastrointestinal symptoms, and sweating. In the majority of them, AEs were limited to the first-second course and categorized as grade 1-2.

In the Phase I-II study by Coiffier, ${ }^{33}$ maximum ofatumumab tolerated dose was not reached. In 27 patients, 246 AEs were registered and almost all categorized as grade 1 or 2 . All patients but one were able to complete treatment. In this last case, acute cytolytic hepatitis considered related to the study drug led to treatment discontinuation after the first infusion. One fatal AE was reported and it was due to one of the four (12\%) grade 3-4 infections reported.

Notably, 3\% of patients developed B-hepatitis reactivation (HBre). Both patients presenting with hepatitis B surface antigen and antibody to hepatitis B core antigen who received rituximab or ofatumumab, were more susceptible to HBre. The rate of $\mathrm{HBre}$ following anti-CD20 antibody has been reported at $16.9 \%$, and seroreversion rate of $20 \%-40 \%$. The majority occurred up to 12 months after cessation of B-cell depleting drugs indicating the potency of the immunosuppressive effect of this drug class and the prolonged immune reconstitution phase. Taking into account these potentially fatal complications, in 2013 the FDA issued a Drug Safety Communication in order to advise clinicians to closely monitor and administer anti-viral prophylaxis to those patients presenting with hepatitis B surface antigen and antibody to hepatitis B core antigen. ${ }^{59}$

Infusion reactions are the most common AE; Wierda et $\mathrm{al}^{34}$ reported $43 \%$ infusion reaction during the first ofatumumab administration decreasing to $6 \%$ at the last one.

Regarding hematologic toxicity, five (15\%) patients developed cytopenia during the dose finding study (thrombocytopenia in three cases, neutropenia in two). No cases of thrombocytopenia were reported by Wierda et $\mathrm{al}^{34}$ at the interim analysis, while in total $13(20 \%)$ patients presented with grade 3-4 neutropenia.

One patient in each of the above mentioned studies developed hemolytic anemia. It is worth noting that in both cases, patients were previously treated with fludarabine.

In the interim analysis from Wierda et al, ${ }^{34}$ infections were also commonly reported in $67 \%$ of patients with $26 \%$ of these categorized as grade 3-4 and leading to death in eight cases. As well as with rituximab, ${ }^{60}$ one case of progressive multifocal leukoencephalopathy was described with ofatumumab. No formation of anti-ofatumumab antibodies was noticed.

Infusion reactions occurred even during retreatment and maintenance, as GEN416 study ${ }^{57}$ reported infusion related AEs in $72 \%$ of patients. Also in this setting most of those were limited to the first or second administration. Nearly one third of patients had an infection leading to death, in three cases due to pneumonia. Infection rate was comparable to the one reported in the original trial.

Nevertheless, the use of ofatumumab as exclusive maintenance therapy did not demonstrate additional toxicity as the rate of overall AEs, infections, and death did not significantly differ from observation group in the PROLONG study. ${ }^{58}$

The addition of ofatumumab to bendamustine, did not translate to unexpected toxicity as reported by Cortelezzi et al. ${ }^{37}$ Infusion-related AEs were common during the first infusion, none of these reaching grade 4 . Nearly $80 \%$ of patients experienced at least one grade 3-4 hematological AE, neutropenia being the most common. Three patients died during study treatment, two following an infection.

In a smaller study with ten patients, even using the same ofatumumab-bendamustine combination, Ujiani et al ${ }^{39}$ could 
not reproduce the same tolerability. The same schedule of bendamustine and ofatumumab in this case led to a premature discontinuation of the study because of an excess of unexpected toxicity comprising infusion-related reactions, infection, and neurotoxicity.

In addition to infusion reactions, grade 3-5 infections were described in 33\% of patients treated with DMS and ofatumumab. ${ }^{41}$ This probably reflected the high immunosuppression given by the addition of steroids and anti-CD20 antibody in a setting of pretreated fragile patients.

Obviously, the same good tolerability reported in pretreated patients, was reproduced with treatment-naïve patients.

Even when added to purine analog-based combinations, ofatumumab did not increase treatment toxicity. This was particularly evident in the O-FC study, ${ }^{53}$ where the two cohorts of patients receiving two different ofatumumab doses, did not show difference in terms of hematologic toxicity or need for dose reduction/treatment discontinuation.

Moreover the anti-CD20 antibody was shown to be well tolerated even in the specific setting of elderly and/ or unfit. ${ }^{50,55,56}$ Except for infusion reaction, ofatumumab is feasible even in frail patients and chemo-immunotherapy in COMPLEMENT 1 showed to be comparable to chlorambucil as monotherapy in terms of grade 3-4 events.

It is important to highlight that the results of ofatumumab offered to a "non-trial-selected", "real life" population, confirmed the excellent tolerability and manageability of this MoAb. ${ }^{4-46}$ Nevertheless, also in the series provided by ERIC observational study, ${ }^{44}$ 2/103 patients developed progressive multifocal leukoencephalopathy diagnosed at 6 and 11 months after starting therapy. Both cases received extensive prior therapy including fludarabine, rituximab, and alemtuzumab. As previously reported in literature, ${ }^{60-62}$ the use of ofatumumab in the setting of significant immunodeficiency can be more easily associated with such an uncommon neurological manifestation.

Overall it is reasonable to think that the evidence of infections with ofatumumab could be mainly correlated with the condition of a heavily pretreated disease itself, mostly with highly immunosuppressive agents, as all the series included patients with two or more previous lines of therapy.

\section{Discussion}

A retrospective single-institution analysis conducted by MDACC, focused on the poor outcome of double or BF-patients. Patients treated with different salvage approaches, including MoAb, single-agent cytotoxic drugs or intensive chemotherapy combination, obtained a $23 \%$ response rate. Thirteen percent of early deaths and 54\% risk of major infection were described. OS survival result was 9 months. A lack of response and shorter survival (6 months median) was observed in those receiving a chemo-free regimen. ${ }^{62}$ Taking into account historical results, ofatumumab as a single agent definitely showed satisfactory responses in this subset. Moreover, ofatumumab's feasibility and low toxicity rate was underlined by almost all the trials employing this MoAb, as well as in the daily-life setting. Furthermore, it is important to stress the activity of ofatumumab on constitutional-symptom improvement even in those cases not presenting an objective response. ${ }^{34}$ This of course translates in a gain in patients' daily-life quality. Nevertheless, at the last American Society of Hematology meeting, Österborg et al presented the results from the Phase III study OMB1 14242. ${ }^{26}$ One hundred and twenty patients with bulky fludarabine disease, were randomly assigned to ofatumumab (79 cases) vs physician's choice therapy (43 cases). Ofatumumab as salvage treatment, in this selected high-risk population, obtained a $37 \%$ ORR, a slightly inferior result than the pivotal trials. Furthermore, ofatumumab did not meet the primary end point of the study, not being able to demonstrate a clear superiority in respect to best available therapies in terms of PFS (5.4 months with ofatumumab vs 3.6 months with physician's choice).

The lack of a satisfactory prolongation of PFS and OS, represents the most evident single agent ofatumumab limitation.

In the setting of previously treated cases, ofatumumab used as monotherapy showed a significant number of responses but limited PFS ranging between 5 and 11 months (Table 2). Certainly, the population considered in the different studies was selected among those with very few treatment options. Moreover, in most of the above studies, patients received an extensive number of prior therapies before ofatumumab; so probably patients included in these trials were more likely to carry or have acquired adverse biological features.

The advent of new targeted therapies offered a radical change in CLL treatment scenery. The first openlabel Phase III study directly comparing ofatumumab and ibrutinib,${ }^{64}$ showed a clear superiority of the latter in terms of responses and survival. Furthermore, ibrutinib, as well as other small molecules, was able to abrogate the negative impact $17 \mathrm{p}$ deletion. The addition of ofatumumab to the new targeted therapies could represent a promising association. This was recently proved by a Phase $1 b / 2$ study in which ofatumumab combined with ibrutinib, led to a response up 
Table 2 Clinical trials in relapsed/refractory patients

\begin{tabular}{|c|c|c|c|c|c|c|}
\hline Authors & Schedule & $\begin{array}{l}\text { Patient } \\
\text { number }\end{array}$ & $\begin{array}{l}\text { ORR (\%) } \\
\text { (CRR \%) }\end{array}$ & $\begin{array}{l}\text { Follow-up } \\
\text { (mo) }\end{array}$ & PFS (mo) & OS (mo) \\
\hline Coiffier et al $2008^{33}$ & $\begin{array}{l}\text { O: } 100 \mathrm{mg} \rightarrow 500 \mathrm{mg} \\
\text { O: } 300 \mathrm{mg} \rightarrow 1,000 \mathrm{mg} \\
\text { O: } 500 \mathrm{mg} \rightarrow 2,000 \mathrm{mg} \\
\text { For } 4 \text { infusions }\end{array}$ & $\begin{array}{l}3 \\
3 \\
27\end{array}$ & $\begin{array}{l}33 \\
0 \\
50\end{array}$ & NR & $\begin{array}{l}2.6 \\
2.5 \\
4.4\end{array}$ & NR \\
\hline Wierda et al $2010^{34}$ & $\begin{array}{l}\text { O: } 300 \mathrm{mg} \text { DI; } 2,000 \mathrm{mg} \mathrm{D} 8 \\
2,000 \mathrm{mg} \text { weekly for } 6 \mathrm{w} \\
2,000 \mathrm{mg} \text { monthly for } 4 \mathrm{mo} \\
\text { FA-ref } \\
\text { BF-ref }\end{array}$ & $\begin{array}{l}59 \\
79\end{array}$ & $\begin{array}{l}57 \\
48\end{array}$ & NR & $\begin{array}{l}5.7 \\
5.9\end{array}$ & $\begin{array}{l}13.7 \\
15.4\end{array}$ \\
\hline Cortelezzi et al $2014^{37}$ & $\begin{array}{l}\text { O: } 300 \mathrm{mg} \text { CIDI, I,000 mg CID8; } \\
\text { I,000 mg DIC2 } \rightarrow 6 \\
\text { B: } 70 \mathrm{mg} / \mathrm{m}^{2} \mathrm{DI}-2 \mathrm{Cl} \text { to } \mathrm{C} 6\end{array}$ & 47 & 72 ( 17$)$ & 24.2 & $49.6 \%$ & $83.6 \%$ \\
\hline Offner et al $2014^{38}$ & $\begin{array}{l}\text { O: } 300 \mathrm{mg} \text { CIDI, I,000 mg CID8; } \\
\text { I,000 mg DIC2 } \rightarrow 6 \\
\text { B: } 70 \mathrm{mg} / \mathrm{m}^{2} \mathrm{DI}-2 \mathrm{CI} \rightarrow 6\end{array}$ & 53 & $74(I I)$ & 8.7 & $\mathrm{nr}$ & $\mathrm{nr}$ \\
\hline Castro et al $2014^{40}$ & $\begin{array}{l}\text { O: } 300 \mathrm{mg} \mathrm{DI} ; \mathrm{I}, 000 \mathrm{mg} \text { weekly for } 12 \mathrm{w} \\
\mathrm{mPDN} \text { I,000 mg/m² DI-3 q28 }\end{array}$ & 21 & $81(5)$ & 31 & 9.9 & $\mathrm{nr}$ \\
\hline Doubek et al $2015^{41}$ & $\begin{array}{l}\text { O: CIDI: } 300 \mathrm{mg}, \mathrm{D} 8 \\
\text { I5, 22: } 2,000 \mathrm{mg} \\
\text { C2-6 DI, } 8,15,22: 1,000 \mathrm{mg} \\
\text { Dexa DI } \rightarrow 4 ; 15 \rightarrow \mathrm{I} 8 \mathrm{CI} \rightarrow \mathrm{C} 6\end{array}$ & 33 & $67(15)$ & NR & II & 34 \\
\hline Costa et al $2015^{43}$ & $\begin{array}{l}\mathrm{O}: \mathrm{Cl} \rightarrow 62,000 \mathrm{mg} \mathrm{DI} \\
\text { lenalidomide } 10 \mathrm{mg} \mathrm{D} 8 \rightarrow 28 \\
\mathrm{q} 28 \text { for } 6 \mathrm{C}\end{array}$ & 21 & 47.6 & 16.1 & $\mathrm{nr}$ & 21.5 \\
\hline Byrd et al $2014^{64}$ & $\begin{array}{l}\text { O: } 300 \text { mg DI; } 2,000 \text { mg D8; } \\
2,000 \text { mg weekly for } 6 \text { w; } \\
2,000 \text { mg monthly for } 4 \text { mo vs } \\
\text { ibrutinib } 420 \text { mg daily }\end{array}$ & $\begin{array}{l}196 \\
\text { vs } \\
195\end{array}$ & $\begin{array}{l}4(0) \text { vs } \\
43(0)\end{array}$ & 9.4 & $\begin{array}{l}\text { 8.I mo vs } \\
\mathrm{nr}\end{array}$ & $\begin{array}{l}\mathrm{nr} \text { in both (at I } 2 \\
\mathrm{mo} 8 \mathrm{I} \text { vs } 90 \%)\end{array}$ \\
\hline Jaglowski et al $2015^{65}$ & $\begin{array}{l}\text { O: } 300 \text { mg w5; } 2,000 \text { mg w6; } 2,000 \text { mg } \\
\text { weekly for } 6 \text { w; } 2,000 \text { mg monthly for } \\
4 \text { mo + ibrutinib } 420 \text { mg daily from DI } \\
\text { O: starting from wl D2 + } \\
\text { ibrutinib } 420 \mathrm{mg} \text { daily from wI DI } \\
\text { O: starting from wl DI + } \\
\text { ibrutinib } 420 \text { mg daily from w9 }\end{array}$ & $\begin{array}{l}27 \\
20 \\
24\end{array}$ & $\begin{array}{l}100 \\
79 \\
71\end{array}$ & 12.5 & $\begin{array}{l}\mathrm{nr} \text { (at } 12 \text { mo } 88.7 \% \text { ) } \\
\mathrm{nr} \text { (at } 12 \text { mo } 85 \%) \\
\mathrm{nr} \text { (at } 12 \text { mo } 75 \% \text { ) }\end{array}$ & $\begin{array}{l}\mathrm{nr} \text { (at } 12 \text { mo } 92.3 \%) \\
\mathrm{nr}(\text { at } 12 \text { mo } 85 \%) \\
\mathrm{nr} \text { (at I } 2 \text { mo 87.5) }\end{array}$ \\
\hline Moreno et al $2015^{44}$ & Observational & 103 & $22(3)$ & 9.4 & 5 & II \\
\hline Chowdhury et al 20I I 45 & Observational & 27 & $48(I I)$ & 8 & 5.5 (TTNT) & $\mathrm{nr}$ \\
\hline Dupuis et al $2015^{46}$ & Observational & 30 & $47(13)$ & 8 & 4.3 & 8.3 \\
\hline
\end{tabular}

Abbreviations: O, ofatumumab; B, bendamustine; Dexa, dexamethasone; mPDN, methylprednisolone; FA-ref, fludarabine refractory; BF-ref, bulky-fludarabine refractory; ORR, overall response rate; CR, complete response; CRR, complete remission rate; PFS, progression free survival; OS, overall survival; mo, month(s); NR, not reported; nr, not reached; D, day; w, week(s); C, cycle; TTNT, time to next treatment.

to $100 \%, 79 \%$, and $71 \%$ in high-risk CLL patients treated respectively with ibrutinib lead-in (group 1), concurrent start (group 2), and ofatumumab led-in (group 3) schedule. The high ORR was not affected by high risk factors such as $17 \mathrm{p}$ deletion, unmutated IgHV or high B2MG value. Estimated 12 months PFS resulted in $89 \%, 85 \%$, and $75 \%$ of patients respectively. ${ }^{65}$ These results appear to be superior compared to ibrutinib as single agent and comparable to ibrutinib combined with rituximab. ${ }^{66,67}$ The importance of a prompt start of ibrutinib was evidenced by the higher response rate in groups 1 and 2 and the evidence of progression in four patients belonging to group 3 waiting to receive ibrutinib.
Currently, FCR remains the gold-standard treatment for young, fit patients, as in this setting ofatumumab failed to demonstrate a superiority in respect to rituximab. ${ }^{16,53}$ A further analysis of the CLL8 trial investigated the influence of the novel gene mutations in response to FCR. Notably, patients carrying NOTCH1 mutation did not seem to benefit from rituximab addition nor did they have clinical and MRD response, nor prolonged PFS and OS. ${ }^{48}$

The reduced efficacy of rituximab in patients with NOTCH1 mutation has also been proven in the setting of consolidation/maintenance therapy. Similarly to that previously reported, NOTCH1 mutation was predictive of 
a reduced response rate, response duration, and OS after prolonged rituximab therapy when compared with NOTCH1 wild-type patients. ${ }^{68}$

This clinical resistance has been recently demonstrated also in vitro. In a subset of NOTCH1 mutated CLL cases, cells carrying the mutation showed lower CD20 levels, in part due to histone deacetylase-dependent repression mechanisms. As expected, rituximab CDC-induced lysis was reduced. ${ }^{69}$

Taking into account that complement dependent cytotoxicity, as with rituximab, represents the main ofatumumab mechanism of action, an inferior ofatumumab activity has also been demonstrated in NOTCH1 mutated patients in the COMPLEMENT 1 study. In fact, despite the clear superiority of ofatumumab plus chlorambucil in NOTCH1 wild-type patients, those cases carrying the mutation were associated with a shorter PFS exclusively in the chemo-immunotherapy arm, with no impact on PFS in single agent chlorambucil treatment. ${ }^{70}$ This evidence, suggesting the role of NOTCH1 mutation as a predictive marker of a reduced ofatumumab advantage, should guide its rational use.

It is noteworthy that, although most CLL patients are represented by subjects older than 65 years, there is still a significant lack of treatment options for this population. The good tolerability of ofatumumab used both alone or in combination, allowed its use in elderly fit and unfit patients. In these subsets indeed, ofatumumab was able to overcome rituximab-based combination in terms of response quality and survival. Even MRD negativity, which represents a desirable goal in fit patients treated with intensive therapies, became an achievable target following ofatumumab-based combinations. ${ }^{55,56}$ However, the role of obinutuzumab is also emerging. Obinutuzumab (but not rituximab) combined with chlorambucil, demonstrated a survival advantage compared to chlorambucil alone. ${ }^{71}$ The same result was not obtained with the addition of ofatumumab to chlorambucil. ${ }^{56}$ In this series indeed, chemo-immunotherapy did not confer a survival advantage in respect to chlorambucil alone. Direct-comparison studies will be required to demonstrate a clinical advance of one MoAb over the others.

Lastly, up to now the role of ofatumumab as maintenance therapy is still not clear. A longer follow-up will be essential to better define if a real benefit could be added by the use of ofatumumab in this setting.

\section{Conclusion}

The efficacy of ofatumumab in vivo seemed to be quite disappointing when compared to its in vitro properties; this notwithstanding, there is still a large scope for its use. Considering that most of the clinical trials employing ofatumumab have been applied in the setting of high risk CLL, the replacement of this MoAb in a different treatment time, such as earlier disease phases or front line, would seem a reasonable option. Furthermore, taking into account the data concerning ofatumumab activity in vitro and in vivo, ${ }^{46,72,73}$ its use in association with new targeted therapies could represent a promising scenario in those patients with p53 abnormalities. Therefore, more studies would be advisable to explore such combinations in previously treated or untreated $17 \mathrm{p}$ deleted patients.

Further prospective and randomized studies are required to better clarify ofatumumab's ability in combination treatments for untreated patients, maintenance therapy, or at an earlier stage in the course of the disease.

\section{Disclosure}

The authors report no conflicts of interest.

\section{References}

1. National Cancer Institute. Median Age of Cancer Patients at Diagnosis by Primary Cancer Site, Race and Sex, 2003-2007. Bethesda, MD: National Cancer Institute; 2010. Available from: http://seer.cancer. gov/archive/csr/1975_2007/results_single/sect_01_table.11_2pgs.pdf. Accessed August 3, 2015.

2. Lundin J, Kimby E, Björkholm M, et al. Phase II trial of subcutaneous anti-CD52 monoclonal antibody alemtuzumab (Campath-1H) as firstline treatment for patients with B-cell chronic lymphocytic leukemia (B-CLL). Blood. 2002;100(3):768-773.

3. Stilgenbauer S, Zenz T, Winkler D, et al. Subcutaneous alemtuzumab in fludarabine-refractory chronic lymphocytic leukemia: clinical results and prognostic marker analyses from the CLL2H study of the German Chronic Lymphocytic Leukemia Study Group. J Clin Oncol. 2009;27(24):3994-4001.

4. Hillmen P, Skotnicki A, Robak T, et al. Alemtuzumab compared with chlorambucil as first-line therapy for chronic lymphocytic leukemia. J Clin Oncol. 2007;25(35):5616-5623.

5. Keating MJ, Flinn I, Jain V, et al. Therapeutic role of alemtuzumab (Campath-1H) in patients who have failed fludarabine: results of a large international study. Blood. 2002;99(10):3554-3561.

6. Glennie MJ, French RR, Cragg MS, Taylor RP. Mechanisms of killing by anti-CD20 monoclonal antibodies. Mol Immunol. 2007;44(16): 3823-3837.

7. Weiner GJ. Rituximab: mechanism of action. Semin Hematol. 2010; 47(2):115-123.

8. Golay J, Lazzari M, Facchintti V, et al. CD20 levels determine the in vivo susceptibility to rituximab and complement of B-cell chronic lymphocytic leukemia: further regulation by CD55 and CD59. Blood. 2001;98(12):3383-3389.

9. Montserrat E. Rituximab in chronic lymphocytic leukemia. Semin Oncol. 2003;30(1 Suppl 2):34-39.

10. Huhn D, von Schilling C, Wilhelm M, et al. Rituximab therapy of patients with B-cell chronic lymphocytic leukemia. Blood. 2001;98(5): 1326-1331.

11. Hainsworth J, Litchy S, Baron J, et al. Single agent rituximab as first line and maintenance treatment for patients with chronic lymphocytic leukemia or small lymphocytic lymphoma: a phase II trial of the Minnie earl Cancer Research Network. J Clin Oncol. 2003;21(9):1746-1751. 
12. Vose JM, Link BK, Grossbard ML, et al. Phase II study of rituximab in combination with chop chemotherapy in patients with previously untreated, aggressive non-Hodgkin's lymphoma. J Clin Oncol. 2001; 19(2):389-397.

13. Coiffier B, Haioun C, Ketterer N, et al. Rituximab (anti-CD20 monoclonal antibody) for the treatment of patients with relapsing or refractory aggressive lymphoma: a multicenter phase II study. Blood. 1998; 92(6):1927-1932.

14. Schulz H, Bohlius JF, Trelle S, et al. Immunochemotherapy with rituximab and overall survival in patients with indolent or mantle cell lymphoma: a systematic review and meta-analysis. J Natl Cancer Inst. 2007;99(9):706-714.

15. Wierda W, O’Brien S, Wen S, et al. Chemoimmunotherapy with fludarabine, cyclophosphamide, and rituximab for relapsed and refractory chronic lymphocytic leukemia. J Clin Oncol. 2005;23(18):4070-4078.

16. Hallek M, Fischer K, Fingerle-Rowson G, et al. Addition of rituximab to fludarabine and cyclophosphamide in patients with chronic lymphocytic leukaemia: a randomised, open-label, phase 3 trial. Lancet. 2010; 376(9747):1164-1174

17. Hillmen P, Gribben JG, Follows GA, et al. Rituximab plus chlorambucil as first-line treatment for chronic lymphocytic leukemia: Final analysis of an open-label phase II study. J Clin Oncol. 2014;32(12):1236-1241.

18. Foà R, Del Giudice I, Cuneo A, et al. Chlorambucil plus rituximab with or without maintenance rituximab as first-line treatment for elderly chronic lymphocytic leukemia patients. Am J Hematol. 2014;89(5):480-486.

19. Kay NE, Geyer SM, Call TG, et al. Combination chemoimmunotherapy with pentostatin, cyclophosphamide, and rituximab shows significant clinical activity with low accompanying toxicity in previously untreated B chronic lymphocytic leukemia. Blood. 2007;109(2):405-411.

20. Shanafelt TD, Lin T, Geyer SM, et al. Pentostatin, cyclophosphamide, and rituximab regimen in older patients with chronic lymphocytic leukemia. Cancer. 2007;109(11):2291-2298.

21. Bertazzoni P, Rabascio C, Gigli F, et al. Rituximab and subcutaneous cladribine in chronic lymphocytic leukemia for newly diagnosed and relapsed patients. Leuk Lymphoma. 2010;51(8):1485-1493.

22. Fischer K, Cramer P, Busch R, et al. Bendamustine in combination with rituximab for previously untreated patients with chronic lymphocytic leukemia: A multicenter phase II trial of the German Chronic Lymphocytic Leukemia Study Group. J Clin Oncol. 2012;30(26):3209-3216.

23. Laurenti L, Autore F, Innocenti I, et al. Bendamustine with Rituximab Is Safe and Effective As FRONT LINE Therapy in Elderly B-CLL Patients. An ITALIAN RETROSPECTIVE MULTICENTER Experience. Abstract presented at: ASH Annual Meeting; December 5-8, 2014; San Francisco, CA.

24. Zenz T, Gribben JG, Hallek M, et al. Risk categories and refractory CLL in the era of chemoimmunotherapy. Blood. 2012;119(18):4101-4107.

25. Keating MJ, O'Brien S, Kontoyiannis D, et al. Results of first salvage therapy for patients refractory to a fludarabine regimen in chronic lymphocytic leukemia. Leuk Lymphoma. 2002;43(9):1755-1762.

26. Tam CS, O'Brien S, Lerner S, et al. The natural history of fludarabinerefractory chronic lymphocytic leukemia patients who fail alemtuzumab or have bulky lymphadenopathy. Leuk Lymphoma. 2007; 48(10):1931-1939.

27. Teeling JL, French RR, Cragg MS, et al. Characterization of new human CD20 monoclonal antibodies with potent cytolytic activity against nonHodgkin lymphomas. Blood. 2004;104(6):1793-1800.

28. Bologna L, Gotti E, Da Roit F, et al. Ofatumumab is more efficient than rituximab in lysing B Chronic Lymphoccitic Leukemia cells in whole blood and in combination with chemotherapy. J Immunol. 2013 190(1):231-239.

29. Okroj M, Eriksson I, Osterborg A, Blom AM. Killing of CLL and NHL cells by rituximab and ofatumumab under limited availability of complement. Med Oncol. 2013;30(4):759.

30. Pawliczkowycz AW, Beurskens FJ, Beum PV, et al. Binding of submaximal C1q promotes complement-dependent cytotoxicity (CDC) of B cells opsonized with anti-CD20 mAbs ofatumumab (OFA) or rituximab (RTX): considerably higher levels of CDC are induced by OFA than by RTX. J Immunol. 2009;183(1):749-758.
31. Shimada K, Tomita A, Saito S, Kiyoi H. Efficacy of ofatumumab against rituximab-resistant B-CLL/SLL cells with low CD20 protein expression. Br J Haematol. 2014;166(3):455-457.

32. Rai KR, Freter CE, Mercier RJ, et al. Alemtuzumab in previously treated chronic lymphocytic leukemia patients who also had received fludarabine. J Clin Oncol. 2002;20(18):3891-3897.

33. Coiffier B, Lepretre S, Pedersen LM, et al. Safety and efficacy of ofatumumab, a fully human monoclonal anti-CD20 antibody, in patients with relapsed or refractory B-cell chronic lymphocytic leukemia: a phase 1-2 study. Blood. 2008;111(3):1094-1100.

34. Wierda WG, Kipps TJ, Mayer J, et al. Ofatumumab as a single-agent CD20 immunotherapy in fludarabine-refractory chronic lymphocytic leukemia. J Clin Oncol. 2010;28(10):1749-1755.

35. Österborg A, Jewell RC, Padmanabhan-Iye S, et al. Ofatumumab monotherapy in fludarabine-refractory chronic lymphocytic leukemia: final results from a pivotal study. Haematologica. Epub 2015 Mar 13.

36. Wierda WG, Padmanabhan S, Chan GW, et al. Ofatumumab is active in fludarabine-refractory CLL irrespective of prior rituximab: results from the phase 2 international study. Blood. 2011;118(19):5126-5129.

37. Cortelezzi A, Sciumè M, Liberati AM, et al. Bendamustine in combination with ofatumumab in relapsed or refractory chronic lymphocytic leukemia: a GIMEMA Multicenter Phase II Trial. Leukemia. 2014; 28(3):642-648.

38. Offner F, Panagiotidis P, Afanasyev B, et al. Ofatumumab therapy in patients with untreated and relapsed chronic lymphocytic leukemia: initial results of the phase II study OMB115991. Presented at: ASH Meeting; December 5, 2014; San Francisco, CA, USA.

39. Ujjani C, Ramzi P, Gehan E, Wang H, Wang Y, Cheson BD. Ofatumumab and bendamustine in previously treated chronic lymphocytic leukemia and small lymphocytic lymphoma. Leuk Lymphoma. 2015;56(4):915-920.

40. Castro JE, Choi MY, Carvajal T, et al. Ofatumumab and high-dose methylprednisolone for the treatment of patients with relapsed or refractory chronic lymphocytic leukemia. Blood Cancer J. 2014;4:e258.

41. Doubek M, Brychtova Y, Panovska A, et al. Ofatumumab added to dexamethasone in patients with relapsed or refractory chronic lymphocytic leukemia: Results from a phase II study. Am J Hematol. 2015; 90(5):417-421.

42. Wu L, Adams M, Carter T, et al. Lenalidomide enhances natural killer cell and monocyte-mediated antibody-dependent cellular cytotoxicity of rituximab-treated CD20+ tumor cells. Clin Cancer Res. 2008;14(14):4650-4657.

43. Costa LJ, Fanning SR, Stephenson J Jr, et al. Sequential ofatumumab and lenalidomide for the treatment of relapsed and refractory chronic lymphocytic leukemia and small lymphocytic lymphoma. Leuk Lymphoma. 2015;56(3):645-649.

44. Moreno C, Montillo M, Panayiotidis P, et al. Ofatumumab in poorprognosis chronic lymphocytic leukemia: a Phase IV, non-interventional, observational study from the European Research Initiative on Chronic Lymphocytic Leukemia. Haematologica. 2015;100(4):511-516.

45. Chowdhury O, Varghese A, Pattinson J, et al. Ofatumumab in advanced stage chronic lymphocytic leukaemia: results of the UK named patient compassionate use programme. Br J Haematol. 2011; 155(4):519-521.

46. Dupuis J, Brice P, François S, et al. Ofatumumab in refractory chronic lymphocytic leukemia: experience through the French early access program. Clin Lymphoma Myeloma Leuk. 2015;15(2):43-46.

47. Strati P, Wierda W, Burger J, et al. Myelosuppression after frontline fludarabine, cyclophosphamide, and rituximab in patients with chronic lymphocytic leukemia: analysis of persistent and new-onset cytopenia. Cancer. 2013;119(21):3805-3811.

48. Stilgenbauer S, Schnaiter A, Paschka P, et al. Gene mutations and treatment outcome in chronic lymphocytic leukemia: results from the CLL8 trial. Blood. 2014;123(21):3247-3254.

49. Flinn IW, Harwin WN, Macias-Pérez IM, et al. A Phase II Trial of Ofatumumab for Older Patients and Patients Who Refuse Fludarabine-Based Regimens with Previously Untreated Chronic Lymphocytic Leukemia or Small Lymphocytic Lymphoma. Abstract presented at: ASH Annual Meeting; December 8-11, 2012; Atlanta, GA. 
50. Ciccone M, Vitale C, Hinojosa C, et al. Early Results of a Phase II Study of Ofatumumab As Front-Line Treatment in Elderly, Unfit Patients with Chronic Lymphocytic Leukemia (CLL). Abstract presented at: ASH Annual Meeting; December 5-8, 2014; San Francisco, CA.

51. Jain N, Keating MJ, Ferrajoli A, et al. Early Ofatumumab Treatment For High-Risk, Treatment-Naive Patients With Chronic Lymphocytic Leukemia. Abstract presented at: ASH Annual Meeting; December 7-10, 2013; New Orleans, LA.

52. Ma S, Rosen S, Frankfurt O, et al. A Phase 2 Study of AlemtuzumabOfatumumab (A+O) Combination in Patients with Previously Untreated Chronic Lymphocytic Leukemia (CLL). Abstract presented at: ASH Annual Meeting; December 5-8, 2014; San Francisco, CA.

53. Wierda WG, Kipps TJ, Dürig J, et al. Chemoimmunotherapy with O-FC in previously untreated patients with chronic lymphocytic leukemia. Blood. 2011;117(24):6450-6458.

54. Shanafelt T, Lanasa MC, Call TG, et al. Ofatumumab-based chemoimmunotherapy is effective and well tolerated in patients with previously untreated chronic lymphocytic leukemia (CLL). Cancer. 2013; 119(21):3788-3796.

55. Montillo M, Rossi D, Motta M, et al. A Phase II Multi-Center Trial Of Pentostatin Plus Cyclophosphamide With Ofatumumab (PCO) In Older Previously Untreated Chronic Lymphocytic Leukemia (CLL) Patients. Abstract presented at: ASH Annual Meeting; December 7-10, 2013; New Orleans, LA.

56. Hillmen P, Robak T, Janssens A, et al. Chlorambucil plus ofatumumab versus chlorambucil alone in previously untreated patients with chronic lymphocytic leukaemia (COMPLEMENT 1): a randomised, multicentre, open-label phase 3 trial. Lancet. 2015;385(9980):1873-1883.

57. Österborg A, Wierda WG, Mayer J, et al. Ofatumumab retreatment and maintenance in fludarabine-refractory chronic lymphocytic leukaemia patients. Br J Haematol. 2015;170(1):40-49.

58. van Oers MHJ, Kuliczkowski K, Smolej L, et al. Ofatumumab (OFA) Maintenance Prolongs PFS in Relapsed CLL: Prolong Study Interim Analysis Results. Abstract presented at: ASH Annual Meeting; December 5-8, 2014; San Francisco, CA.

59. Pattullo V. Hepatitis B reactivation in the setting of chemotherapy and immunosuppression - prevention is better than cure. World J Hepatol. 2015;7(7):954-967.

60. Carson KR, Evens AM, Richey EA, et al. Progressive multifocal leukoencephalopathy after rituximab therapy in HIV-negative patients: a report of 57 cases from the research on adverse drug events and reports project. Blood. 2009;113(20):4834-4840.

61. Bruce DR, Rane NS, Schuh A. Progressive multifocal leukoencephalopathy as the presenting feature of chronic lymphocytic leukaemia. Br J Haematol. 2014;167(4):570-571.
62. Isidoro L, Pires P, Rito L, Cordeiro G. Progressive multifocal leukoencephalopathy in a patient with chronic lymphocytic leukaemia treated with alemtuzumab. BMJ Case Rep. 2014;2014. pii: bcr2013201781.

63. Österborg A, Udvardy M, Zaritskey A, et al. Ofatumumab (OFA) Vs. Physician's Choice (PC) of Therapy in Patients (pts) with Bulky Fludarabine Refractory (BFR) Chronic Lymphocytic Leukaemia (CLL): Results of the Phase III Study OMB114242. Abstract presented at: ASH Annual Meeting; December 5-8, 2014; San Francisco, CA.

64. Byrd JC, Brown JR, O'Brien S, et al. Ibrutinib versus Ofatumumab in Previously Treated Chronic Lymphoid Leukemia. N Engl J Med. 2014; 371(3):213-223.

65. Jaglowski SM, Jones JA, Nagar V, et al. Safety and activity of BTK inhibitor ibrutinib combined with ofatumumab in chronic lymphocytic leukemia: a phase 1b/2 study. Blood. Epub 2015 Jun 26.

66. Byrd JC, O'Brien S, James DF, et al. Ibrutinib in relapsed chronic lymphocytic leukemia. N Engl J Med. 2013;369(13):1278-1279.

67. Burger JA, Keating MJ, Wierda WG, et al. Safety and activity of ibrutinib plus rituximab for patients with high-risk chronic lymphocytic leukaemia: a single-arm, phase 2 study. Lancet Oncol. 2014; 15(10):1090-1099.

68. Bo MD, Del Principe MI, Pozzo F, et al. NOTCH1 mutations identify a chronic lymphocytic leukemia patient subset with worse prognosis in the setting of a rituximab-based induction and consolidation treatment. Ann Hematol. 2014;93(10):1765-1774.

69. Pozzo F, Bittolo T, Bulian P, et al. NOTCH1 Mutations Are Associated with Low CD20 Expression in Chronic Lymphocytic Leukemia: Evidences for a NOTCH1-Mediated Epigenetic Regulatory Mechanism. Abstract presented at: ASH Annual Meeting; December 5-8, 2014; San Francisco, CA.

70. Tausch E, Beck P, Schlenk RF, et al. NOTCH1 mutation and treatment outcome in CLL patients treated with Chlorambucil (Chl) or Ofatumumab-Chl (O-Chl): Results from the Phase III Study COMPLEMENT 1 (OMB110911). Abstract presented at: ASH Annual Meeting; December 7-10, 2013; New Orleans, CA.

71. Goede V, Fischer K, Busch R, et al. Obinutuzumab plus chlorambucil in patients with CLL and coexisting conditions. N Engl J Med. 2014; 370(12):1101-1110.

72. Namberger K, Weiss L, Krause B, Melchardt T, Greil R. A case of long-term remission with ofatumumab maintenance therapy in multiply relapsed and rituximab-refractory chronic lymphocytic leukaemia with deletion 17p. Eur J Haematol. 2013;90(4):349-350.

73. Sebejova L, Borsky M, Jaskova Z, et al. Distinct in vitro sensitivity of p53-mutated and ATM-mutated chronic lymphocytic leukemia cells to ofatumumab. Exp Hematol. 2014;42(10):867-874.
Biologics: Targets \& Therapy

\section{Publish your work in this journal}

Biologics: Targets \& Therapy is an international, peer-reviewed journal focusing on the patho-physiological rationale for and clinical application of Biologic agents in the management of autoimmune diseases, cancers or other pathologies where a molecular target can be identified. This journal is indexed on PubMed Central, CAS, EMBase, Scopus

\section{Dovepress}

and the Elsevier Bibliographic databases. The manuscript management system is completely online and includes a very quick and fair peerreview system, which is all easy to use. Visit http://www.dovepress. com/testimonials.php to read real quotes from published authors. 